\title{
Analyzing the nonlinear heat transfer equation by AGM
}

\author{
Hadi Mirgolbabaee, Soheil Tahernejad Ledari, Davood Domiri Ganji and Esmail Karimi Valujai \\ Department of Mechanical Engineering, Babol University of Technology,P.O. Box 484, Babol, Iran
}

Received: 22 October 2015, Accepted: 16 March 2016

Published online: 23 January 2017.

\begin{abstract}
In this paper, a novel nonlinear differential equation in the field of heat transfer has been investigated and solved completely by a new method that we called it Akbari-Ganjis Method (AGM). Regarding to the previously published papers, investigating this kind of equations is a very hard project to do and the obtained solution is not accurate and reliable. This issue will be appeared after comparing the obtained solution by Numerical Method or the Exact Solution. Based on the comparison which has been made between the achieved solutions by AGM and Numerical Method (Runge-Kutte 4th), it is possible to indicate that AGM can be successfully applied to various differential equations particularly for difficult ones. Furthermore, It is necessary to mention that a summary of the excellence of this method in comparison with other approaches can be considered as follows: Boundary conditions are required in accordance with order of the differential equation, this approach can create additional new boundary conditions in regard to the own differential equation and its derivatives. Therefore, it is logical to mention which AGM is operational for miscellaneous nonlinear differential equations in comparison with the other methods.
\end{abstract}

Keywords: Akbari-Ganji'sMethod (AGM), nonlinear equation of heat transfer, numerical method.

\section{Introduction}

Since most of the phenomena in our world are essentially nonlinear and hence described by nonlinear equations, there has developed an ever-increasing interest of scientists and engineers in the analytical asymptotic techniques for solving nonlinear problems. All these problems and phenomena are modeled by ordinary or partial differential equations. In this case study, similarity transformation has been used to reduce the governing differential equations into an ordinary non-linear differential equation. Recently, many new numerical techniques have been widely applied to the nonlinear problems. Some of these methods are Perturbation Method (PM) [1], Homotopy Perturbation Method (HPM) [2,3,4,5, 6], Variational Iteration Method (VIM) [7,8,9], Homotopy Analysis Method (HAM)[10,11], Differential Transform Method (DTM) [12] and Adomian Decomposition Method (ADM) [13,14].

The main purpose of this paper is introducing Akbari-Ganji's Method (AGM) $[15,16,17,18]$ as new methods and by comparing it with numerical methods we can precisely conclude that the AGM has high efficiency and accuracy for solving nonlinear problems with high nonlinearity. It is necessary to mention that a summary of the excellence of this method in comparison with the other approaches can be considered as follows: Boundary conditions are needed in accordance with the order of differential equations in the solution procedure, but when the number of boundary conditions is less than the order of the differential equation, this approach can create additional new boundary conditions in regard to the own differential equation and its derivatives. Therefore, it is logical to mention that the AGM is operational for miscellaneous nonlinear differential equations in comparison with the other methods. 
Table 1: Nomenclature

\begin{tabular}{|l|l|}
\hline$A \boldsymbol{G M}$ & Akbari-Ganji's Method \\
\hline $\boldsymbol{T}$ & Temperature \\
\hline $\boldsymbol{V}$ & Volume \\
\hline $\boldsymbol{A}$ & Surface area \\
\hline$\rho$ & Density \\
\hline$\tau$ & time \\
\hline $\boldsymbol{c}$ & Specific heat \\
\hline $\boldsymbol{h}$ & Heat transfer coefficient \\
\hline$\beta$ & Constant parameter \\
\hline$\omega$ & Angular frequency \\
\hline
\end{tabular}

\section{Basic Idea of Akbari-Ganji's method (AGM)}

Boundary conditions and initial conditions are required for analytical methods of each linear and nonlinear differential equation according to the physics of the problem. Therefore, we can solve every differential equation with any degrees. In order to comprehend the given method in this paper, two differential equations governing on engineering processes will be solved in this new manner.

In accordance with the boundary conditions, the general manner of a differential equation is as follows. The nonlinear differential equation of $p$ which is a function of $u$, the parameter $u$ which is a function of $x$ and their derivatives are considered as follows.

$$
P_{k}: f\left(u, u^{\prime}, u^{\prime \prime}, \ldots, u^{m}\right)=0 ; u=u(x)
$$

where $P_{k}$ is the name of the nonlinear differential equation; and $m$ is the order of the derivatives. Boundary conditions:

$$
\left\{\begin{array}{l}
u(x)=u_{0}, u^{\prime}(x)=u_{1}, u^{(m-1)}(x)=u_{m-1} \text { at }: x=0, \\
u(x)=u_{L_{0}}, u^{\prime}(x)=u_{L_{1}}, u^{(m-1)}(x)=u_{L_{m-1}} \text { at }: x=L
\end{array}\right.
$$

To solve the first differential equation with respect to the boundary conditions at $x=L$ in Eq. (2), the series of letters in the nth order with constant coefficients which is the answer of the first differential equation is considered as follows.

$$
u(x)=\sum_{i=0}^{n} a_{i} x^{i}=a_{0}+a_{1} x^{1}+a_{2} x^{2}+\ldots+a_{n} x^{n}
$$

The more precise answer of Eq. (1), the more choice of series sentences from Eq. (3). In applied problems, approximately five or six sentences in the series are enough to solve nonlinear differential equation. In the answer of differential, regarding the series from degree $(n)$, there are $(n+1)$ unknown coefficients which need $(n+1)$ equation to satisfy. In AGM, a total answer with constant coefficients is required in order to solve differential equations. The boundary conditions of Eq. (2) are used to solve a set of equations which consist of $(n+1)$ ones. The boundary conditions are applied to the functions as follow.

(a) The application of the boundary conditions for the answer of differential Eq. (3) is in the form of when $x=0$,

$$
\left\{\begin{array}{c}
u(0)=a_{0}=u_{0}, \\
u^{\prime}(0)=a_{1}=u_{1} \\
u^{\prime \prime}(0)=a_{2}=u_{2} \\
\vdots \quad \vdots \quad \vdots
\end{array}\right.
$$


When $x=L$,

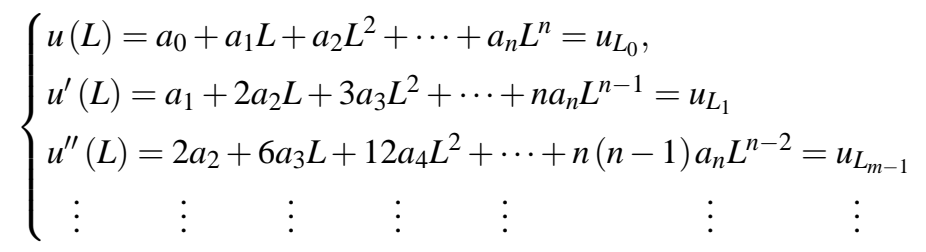

(b) After substituting Eq. (3) into Eq. (1), the application of the boundary conditions on differential Eq. (1) is done according to the following procedure:

$$
\begin{array}{ll}
p_{0}: & f\left(u(0), u^{\prime}(0), u^{\prime \prime}(0), \cdots, u^{(m)}(0)\right) \\
p_{1}: & f\left(u(L), u^{\prime}(L), u^{\prime \prime}(L), \cdots, u^{(m)}(L)\right)
\end{array}
$$

With regard to the choice of $\mathrm{n} ;(n<m)$ sentence in Eq. (3) and in order to make a set of equation which are consisted of $(n+1)$ equations and $(n+1)$ unknowns, we confront with a number of additional unknowns which are certainly the same coefficients of Eq. (3), therefore, to remove this problem, we should make m time derivation in Eq. (1) according to the additional unknowns in the aforementioned set of differential equation. This is the time to apply the boundary condition of Eq. (2).

$$
\begin{array}{ll}
p_{k}^{\prime}: & f\left(u^{\prime}, u^{\prime \prime}, u^{\prime \prime \prime}, \cdots, u^{(m+1)}\right) \\
p_{k}^{\prime \prime}: & f\left(u^{\prime \prime}, u^{\prime \prime \prime}, u^{(I V)}, \cdots, u^{(m+2)}\right)
\end{array}
$$

(c) Application of the boundary conditions on the derivatives of the differential equation $P_{k}$ in Eq. (7) is done in the form of,

$$
\begin{aligned}
& p_{k}^{\prime}:\left\{\begin{array}{l}
f\left(u^{\prime}(0), u^{\prime \prime}(0), u^{\prime \prime \prime}(0), \cdots, u^{(m+1)}(0)\right) \\
f\left(u^{\prime}(L), u^{\prime \prime}(L), u^{\prime \prime \prime}(L), \cdots, u^{(m+1)}(L)\right)
\end{array}\right. \\
& p_{k}^{\prime \prime}:\left\{\begin{array}{l}
f\left(u^{\prime \prime}(0), u^{\prime \prime \prime}(0), \cdots, u^{(m+2)}(0)\right) \\
f\left(u^{\prime \prime}(L), u^{\prime \prime \prime}(L), \cdots, u^{(m+2)}(L)\right)
\end{array}\right.
\end{aligned}
$$

$(n+1)$ equations can be made from Eq. (4) to (9), so that $(n+1)$ unknown coefficients of Eq. (3) will be calculated for example $a_{0}, a_{1}, a_{2} a_{n}$. The answer of the nonlinear differential Eq. (1) will be gained by determining the coefficients of Eq. (3).

\section{Cooling of a lumped system with variable specific heat}

Consider the cooling of a lumped system [19]. Let the system have volume $V$, surface area $A$, density, specific heat $c$ and initial temperature $T_{i}$. At time $t=0$, the system is exposed to a convective environment at temperature $T_{a}$ with convective heat transfer coefficient $h$. Assume that the specific heat $c$ is a linear function temperature of the form,

$$
C=C_{a}\left[1+\beta\left(T-T_{a}\right)\right]
$$


where $C_{a}$ is the specific heat, at temperature $T_{a}$ and $\beta$ is a constant. The cooling equation and the initial condition are

$$
\rho V C \frac{d T}{d t}+h A\left(T-T_{a}\right)=0, T(0)=T_{i}
$$

Introducing Eq. (10) and using the dimensionless parameters,

$$
\theta=\frac{T-T_{a}}{T_{i}-T_{a}}, \quad \tau=\frac{t}{\rho V C_{a} /(h A)}, \quad \varepsilon=\beta\left(T-T_{a}\right) .
$$

Transforms Eq. (11) to

$$
(1+\varepsilon \theta) \frac{d \theta}{d \tau}+\theta=0, \quad \theta(0)=1 .
$$

\subsection{Solving the nonlinear equation by AGM}

First of all we rewrite the problem Eq. (13) in the following order

$$
(1+\varepsilon \theta) \frac{d \theta}{d \tau}+\theta=0
$$

In AGM, the answer of the differential equation is considered as a finite series of polynomials with constant coefficients, as follows

$$
u(\tau)=\sum_{i=0}^{6} a_{i} \tau^{i}=a_{6} \tau^{6}+a_{5} \tau^{5}+a_{4} \tau^{4}+a_{3} \tau^{3}+a_{2} \tau^{2}+a_{1} \tau^{1}+a_{0} .
$$

It is notable that in the aforementioned equation, the constant coefficients $a_{0}$ to $a_{8}$ are obtained by applying the introduced boundary conditions. For this part to write the equations which will be obtained through the solving procedure because the equation become prolongation we write the equations with the mentioned physical ailments as the previous part which is

$$
\varepsilon=0.2
$$

\subsection{Applying boundary conditions}

In AGM, the boundary conditions are applied in order to compute constant coefficients of Eq. (15) in two ways as follows

(i) Applying the boundary conditions on Eq. (16) is expressed as follows

$$
\theta=\theta(B . C)
$$

It is notable that $\mathrm{BC}$ is the abbreviation of boundary conditions. According to the above explanations, the boundary conditions are applied on Eq. (15) in the following form

$$
\theta(0)=1 \rightarrow a_{0}=1
$$

(ii) Applying the boundary condition on the main differential equation, which is this case study is Eq. (14), and also on its derivatives is done after substituting Eq. (15) into the main differential equation as follows

$$
g(\theta(\tau)): \rightarrow g(\theta(B . C)), g^{\prime}(\theta(B . C))
$$

So after substituting Eq. (15) which has been considered as the answer of the main differential equation into Eq. (14), the initial conditions are applied on the obtained equation and also on its derivatives on the basis of Eq. (19) 
as follows.

$$
\begin{aligned}
& g\left(\theta^{\prime}(0)\right): \rightarrow a_{1}+0.2 a_{0} a_{1}+a_{0}=0 \\
& g^{\prime}\left(\theta^{\prime}(0)\right): \rightarrow 2 a_{2}+0.2 a_{1}^{2}+0.4 a_{0} a_{2}+a_{1}=0 \\
& g^{\prime \prime}\left(\theta^{\prime}(0)\right): \rightarrow 6 a_{3}+1.2 a_{1} a_{2}+1.2 a_{0} a_{3}+2 a_{2}=0 \\
& g^{\prime \prime \prime}\left(\theta^{\prime}(0)\right): \rightarrow 24 a_{4}+2.4 a_{2}^{2}+4.8 a_{1} a_{3}+4.8 a_{0} a_{4}+6 a_{3}=0 \\
& g^{(4)}\left(\theta^{\prime}(0)\right): \rightarrow 120 a_{5}+24 a_{2} a_{3}+24 a_{1} a_{4}+24 a_{0} a_{5}+24 a_{4}=0 \\
& g^{(5)}\left(\theta^{\prime}(0)\right): \rightarrow 720 a_{6}+72 a_{3}^{2}+144 a_{2} a_{4}+144 a_{1} a_{5}+144 a_{0} a_{6}+120 a_{5}=0 .
\end{aligned}
$$

By solving a set of algebraic equations which is consisted of eight equations with eight unknowns from Eq. (20) to Eq. (25), the constant coefficients of Eq. (15) can easily be gained as follows

$$
\begin{aligned}
& a_{0}=1, a_{1}=-0.83333333, a_{2}=0.2893518519, a_{3}=-0.04018775720 \\
& a_{4}=-0.004186224709, a_{5}=0.002054351014, a_{6}=0.00006729399287 .
\end{aligned}
$$

Eq. (15) which is the solution of the proposed problem is rewritten form of

$$
\begin{aligned}
& \theta(\tau)=0.00006729399287 \tau^{6}+0.002054351014 \tau^{5}-0.004186224709 \tau^{4} \\
& -0.04018775720 \tau^{3}+0.2893518519 \tau^{2}-0.83333333 \tau+1
\end{aligned}
$$

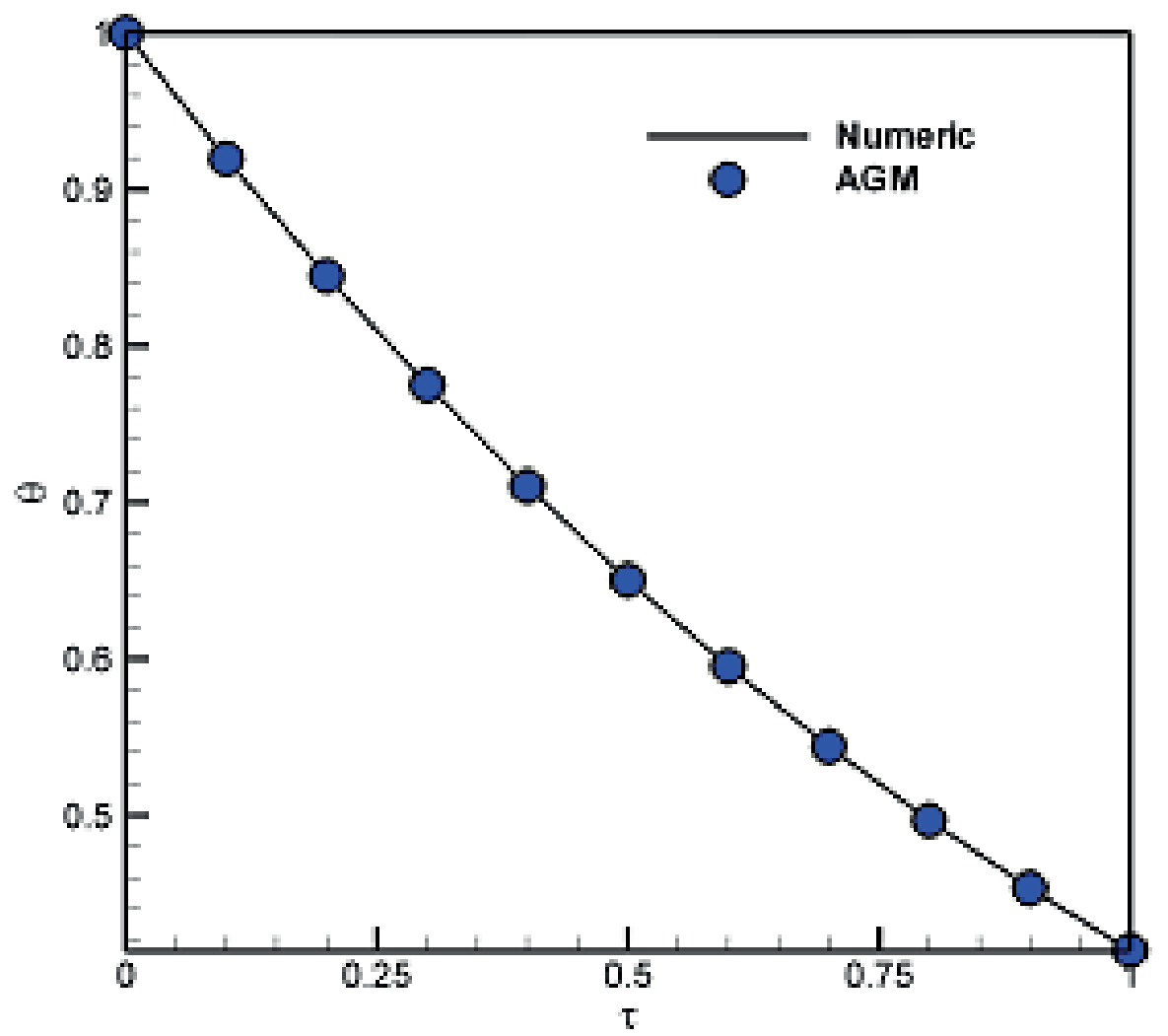

Fig. 1: Comparison of the solution via AGM and exact solution for $\theta(\operatorname{tau})$ at $\varepsilon=0.2$. 
Table 2: A view of Errors of AGM results in comparison Numeric method

\begin{tabular}{|l|l|l|l|}
\hline & Numeric & AGM & Error \\
\hline $\mathbf{0}$ & 1 & 1 & 0 \\
\hline $\mathbf{0 . 1}$ & 0.919519597379591 & 0.9195195994 & 0.0000000021 \\
\hline $\mathbf{0 . 2}$ & 0.844579783987417 & 0.8445798691 & 0.0000001007 \\
\hline $\mathbf{0 . 3}$ & 0.774927625974716 & 0.7749277299 & 0.0000001340 \\
\hline $\mathbf{0 . 4}$ & 0.710304792769913 & 0.7103050914 & 0.0000004203 \\
\hline $\mathbf{0 . 5}$ & 0.650450354716120 & 0.6504514376 & 0.0000016648 \\
\hline $\mathbf{0 . 6}$ & 0.595102981629154 & 0.5951064624 & 0.0000058490 \\
\hline $\mathbf{0 . 7}$ & 0.544002802301944 & 0.5440127527 & 0.0000182910 \\
\hline $\mathbf{0 . 8}$ & 0.496893762198439 & 0.4969185197 & 0.0000498245 \\
\hline $\mathbf{0 . 9}$ & 0.453524788397722 & 0.4535803795 & 0.0001225756 \\
\hline $\mathbf{1}$ & 0.413651800613856 & 0.4137661817 & 0.0002765154 \\
\hline
\end{tabular}

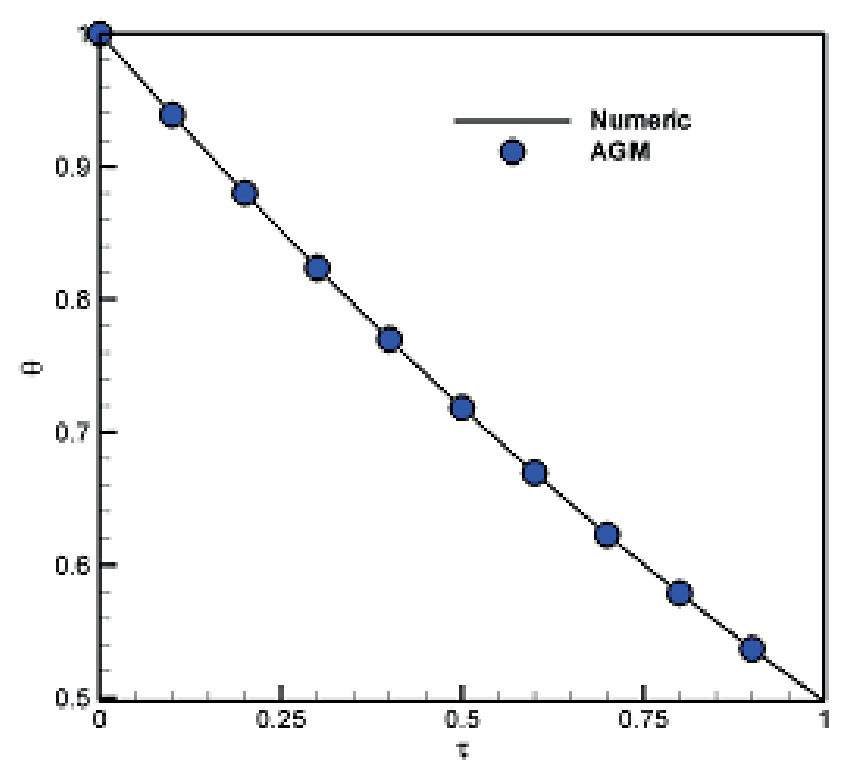

Fig. 2: Comparison of the solution via AGM and exact solution for $\theta(\operatorname{tau})$ at $\varepsilon=0.6$.

\section{Results and discussion}

In Fig. 1 the comparison of the solution between AGM and Exact results is shown and we gained a very interesting agreement between the results is observed, which with consideration of its results error in comparison with exact result from Table.1 it would confirms the excellent validity of the AGM and also in Fig.2 we compared AGM results with Exact solution with different small parameter and we found out the AGM has accurate results for various of small parameter and finally in Fig. 3 we investigated a comparison amongst the obtained $\theta(\operatorname{tau})$ for various of $\varepsilon$.

\section{Conclusion}

In this paper, a new method (AGM) has been proposed for solving a nonlinear differential equation. The afore-mentioned procedures have been done to indicate the ability of AGM for solving differential equation which is shown in the relevant 


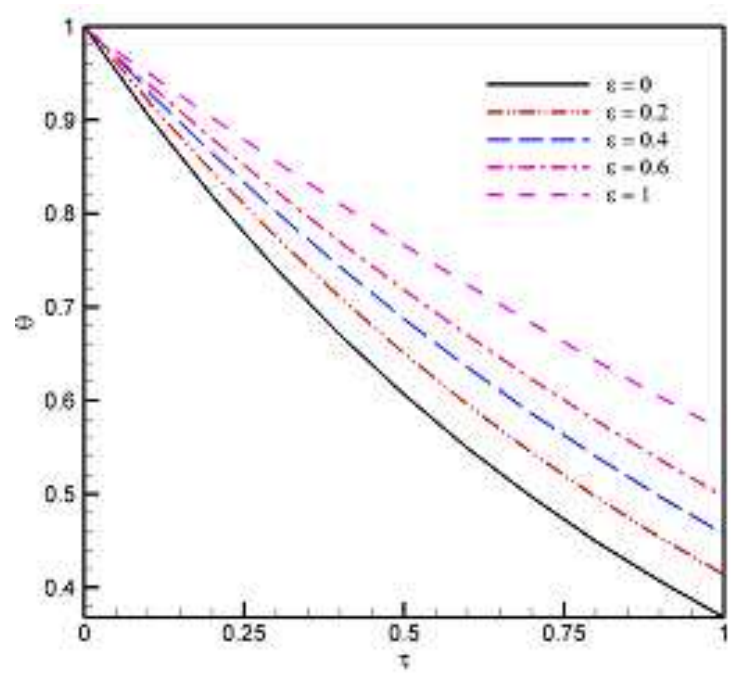

Fig. 3: Comparison of the solution via AGM and exact solution for $\theta(t a u)$ for different $\varepsilon$.

plots and table, we could certainly claim that the obtained answer by this method is close to numerical solution. There are explicit advantages in this approach in comparison to the other semi-analytic methods such as: 1) Differential equations are directly solvable by this method; 2) with regard to the accretion of series sentences, the precision of the solution increases significantly; 3 ) it is not necessary to convert variables into new ones; 4) without any dimensionless procedures, we can solve the problem. In this method, the shortage of boundary condition(s) for solving differential equation(s) is terminated completely. The AGM is operational for miscellaneous nonlinear differential equations which we are hopeful in the near future and will be applied by enthusiastic young researchers.

\section{Competing interests}

The authors declare that they have no competing interests.

\section{Authors' contributions}

All authors have contributed to all parts of the article. All authors read and approved the final manuscript.

\section{References}

[1] D. Ganji, M. Hosseini, and J. Shayegh, "Some nonlinear heat transfer equations solved by three approximate methods," International Communications in Heat and Mass Transfer, vol. 34, pp. 1003-1016, 2007.

[2] M. Sheikholeslami, D.D. Ganji, Heat transfer of Cu-water nanofluid flow between parallel plates, Powder Technology 235 (2013) 873879.

[3] He J. H., Homotopy perturbation technique, Compute. Methods Appl Mech. Eng, 178, 1999, 257262.

[4] M.G. Sfahani, S.S. Ganji, A. Barari, H. Mirgolbabaei, G. Domairry. Analytical solutions to nonlinear conservative oscillator with fifth-order nonlinearity. Earthquake Engineering and Engineering Vibration 9 (3), 367-374.

[5] D.D. Ganji, M. Rafei, A. Sadighi, Z.Z. Ganji, A comparative comparison of He's method with perturbation and numerical methods for nonlinear vibrations equations, Int. J. Nonlinear Dyn. Eng. Sci. 1 (1) (2009) 120. 
[6] M. Gorji, D.D. Ganji, S. Soleimani, New application of He's homotopy perturbation method, Int. J. Nonlinear Sci. Numer. Simul. 8 (3) (2007) 319328.

[7] M. Sheikholeslami, R. Ellahi, H. R. Ashorynejad, G. Domairry, and T. Hayat, Effects of Heat Transfer in Flow of Nanofluids Over a Permeable Stretching Wall in a Porous Medium, Journal of Computational and Theoretical Nanoscience, Vol. 11, 111, 2014.

[8] He J. H., Variational iteration method for autonomous ordinary differential systems, Appl. Math. Compute, 114, $2000,115123$.

[9] S. Ghafoori, M. Motevalli, M. G. Nejad, F. Shakeri, D. D. Ganji, and M. Jalaal, Efficiency of differential transformation method for nonlinear oscillation: Comparison with HPM and VIM, Current Applied Physics, vol. 11, no. 4, pp. 965-971, 2011.

[10] M. Sheikholeslami, R. Ellahi, H. R. Ashorynejad, G. Domairry, and T. Hayat, Effects of Heat Transfer in Flow of Nanofluids Over a Permeable Stretching Wall in a Porous Medium, Journal of Computational and Theoretical Nanoscience, Vol. 11, 111, 2014.

[11] M. Sheikholeslami, H. R. Ashorynejad, G. Domairry and I. Hashim, Flow and Heat Transfer of Cu-Water Nanofluid between a Stretching Sheet and a Porous Surface in a Rotating System, Hindawi Publishing Corporation Journal of Applied Mathematics Volume 2012, Article ID 421320, 19 pages, http://dx.doi.org/10.1155/2012/421320.

[12] M. Sheikholeslami, M.M. Rashidi, Dhafer M. Al Saad,F. Firouzi,Houman B. Rokni, G. Domairry, Steady nanofluid flow between parallel plates considering Thermophoresis and Brownian effects, Journal of King Saud University - Science, (2015),http://dx.doi.org/10.1016/j.jksus.2015.06.003

[13] Adomian G. Solving Frontier Problems of Physics, the Decomposition Method. Boston: Kluwer Academic Publishers, 1994.

[14] M. Sheikholeslami, D.D. Ganji, H.R. Ashorynejad, Investigation of squeezing unsteady nanofluid flow using ADM, Powder Technology 239 (2013) 259265.

[15] S.T. Ledari, H. Mirgolbabaee, D.D. Ganji, Heat transfer analysis of a fin with temperature dependent thermal conductivity and heat transfer coefficient, New Trends in Mathematical Sciences, No. 2, Pages 55-69, 2015.

[16] H. Mirgolbabaee, S.T. Ledari, D.D. Ganji, New approach method for solving Duffing-type nonlinear oscillator, Alexandria Engineering Journal, Volume 55, Issue 2, June 2016, Pages 1695-1702, ISSN 1110-0168, http://dx.doi.org/10.1016/j.aej.2016.03.007.

[17] H. Mirgolbabaee, S.T. Ledari, D.D. Ganji, An assessment of a semi analytical AG method for solving nonlinear oscillators, New Trends in Mathematical Sciences, No. 1, Paper 283-299, 2016, http://dx.doi.org/10.1016/j.aej.2016.03.007.

[18] S.T.Ledari, H. Mirgolbabaee, D.D.Ganji. An assessment of a semi analytical AG method for solving two-dimension nonlinear viscous flow, International Journal of Nonlinear Analysis and Applications, 6(2): 47-64 (2015).

[19] A. Yaziz, G. Hamad, Int. J. Mech. Eng. Educ. 5 (1977) 167. 